\title{
Approximation of large-scale dynamical systems: An overview ${ }^{*}$
}

\author{
A.C. Antoulas ${ }^{\ddagger}$ and D.C. Sorensen ${ }^{\S}$
}

February 7, 2001

\begin{abstract}
In this paper we review the state of affairs in the area of approximation of large-scale systems. We distinguish among three basic categories, namely the SVD-based, the Krylov-based and the SVD-Krylov-based approximation methods. The first two were developed independently of each other and have distinct sets of attributes and drawbacks. The third approach seeks to combine the best attributes of the first two.
\end{abstract}

\section{Contents}

1 Introduction and problem statement 1

2 Motivating Examples 3

3 Approximation methods 4

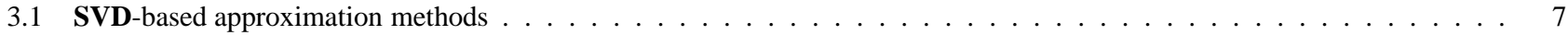

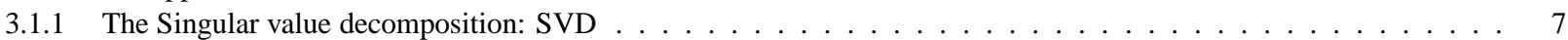

3.1 .2 Proper Orthogonal Decomposition (POD) methods . . . . . . . . . . . . . . . . . . . . . 8

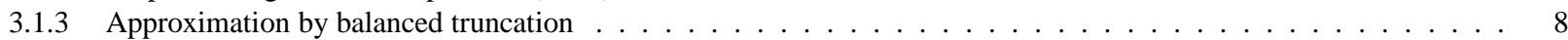

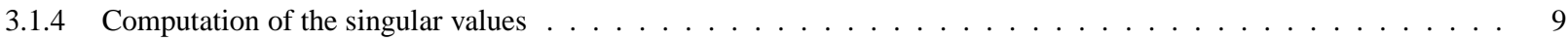

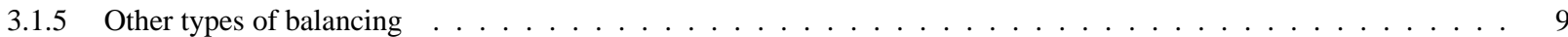

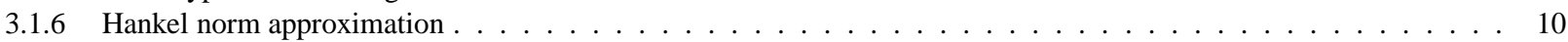

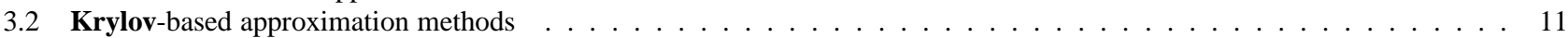

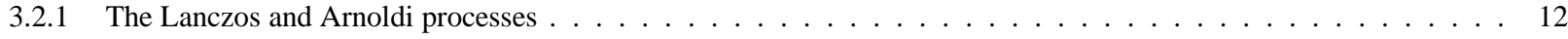

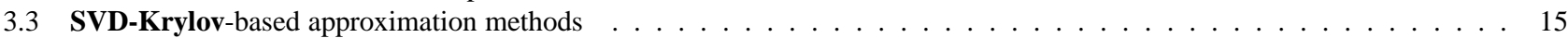

4 Numerical experiments 16

5 Unifying features and conclusions 17

\section{Introduction and problem statement}

Differential equations are one of the most successful means of modeling dynamical systems. In this paper we will deal with the problem of simplification or model reduction of dynamical systems described by differential equations. Model reduction has a long history in the systems and control literature. In fact, the general topic of dimension reduction in dynamical systems is pervasive in the applied mathematics literature. The systems we will

\footnotetext{
${ }^{*}$ This work was supported in part by the NSF Grant DMS-9972591 and by the NSF Grant CCR-9988393.

${ }^{\dagger}$ This paper was presented at the MTNS, Perpignan, June 19-23, 2000.

${ }^{\ddagger}$ Dept. of Electrical and Computer Engineering, Rice University, Houston, TX 77005-1892 (aca@ece.rice.edu)

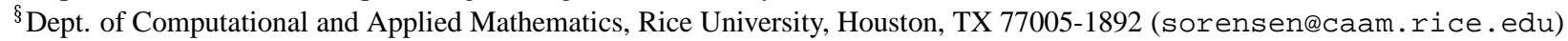


consider will be denoted by $\boldsymbol{\Sigma}$, and will be modeled by means of a set of first order coupled differential equations, together with a set of algebraic equations:

$$
\boldsymbol{\Sigma}:\left\{\begin{array}{l}
\frac{d x(t)}{d t}=f(x(t), u(t)) \\
y(t)=h(x(t), u(t))
\end{array}\right.
$$

For simplicity we will use the following notation:

$$
\boldsymbol{\Sigma}=(f, h), u(t) \in \mathbb{R}^{m}, x(t) \in \mathbb{R}^{n}, y(t) \in \mathbb{R}^{p}
$$

In this setting, $u$ is the input or excitation function, $x$ is the state, and the function $f$ describes the dynamics of $\boldsymbol{\Sigma}$; $y$ on the other hand, is the output or set of observations and $h$ describes the way that the observations are deduced from the state and the input. The complexity of $\boldsymbol{\Sigma}$ is defined as the number of states $n$.

The problem that will be addressed is to simplify or approximate $\boldsymbol{\Sigma}$ with another dynamical system $\hat{\boldsymbol{\Sigma}}$

$$
\hat{\boldsymbol{\Sigma}}=(\hat{f}, \hat{h}), u(t) \in \mathbb{R}^{m}, \hat{x}(t) \in \mathbb{R}^{k}, \hat{y}(t) \in \mathbb{R}^{p}
$$

The first requirement is that the number of states (i.e. the number of first order differential equations) of the approximant be less (or much less) than that of the original system

$$
k \ll n
$$

Obviously in the absence of additional ones, this requirement is easy to satisfy by mere elimination of equations and state variables. The difficulty arises by imposing additional constraints; one set of constraints usually attached to such an approximation problem is the following:

(1) Approximation error small - existence of global error bound

(2) Preservation of stability, passivity

(3) Procedure computationally efficient

(4) Procedure automatic based on error tolerance

The first constraint above is to be understood as follows. Let the input function $u$ be fed into both $\boldsymbol{\Sigma}$ and $\hat{\boldsymbol{\Sigma}}$, and let the corresponding responses be $y$ and $\hat{y}$, respectively. Small approximation error means that $y$ is close to $\hat{y}$ in an appropriately defined sense and for a given set of inputs; in the sequel we will use the following criterion in coming up with reduced-order approximants: the worse error $\|y-\hat{y}\|$ should be minimized for all $u$ (this gives rise to the so-called $\mathcal{H}_{\infty}$ error criterion). In this regard it should be noted that often reduced-order systems are abstract representations which loose physical meaning.

An important special case, is that of linear dynamical systems:

$$
\boldsymbol{\Sigma}:\left\{\begin{array}{l}
\frac{d x(t)}{d t}=A x(t)+B u(t) \\
y(t)=C x(t)+D u(t)
\end{array}\right.
$$

For simplicity we will use the notation:

$$
\boldsymbol{\Sigma}=\left(\begin{array}{l|l}
A & B \\
\hline C & D
\end{array}\right) \in \mathbb{R}^{(n+p) \times(n+m)}
$$


The problem in this case consists in approximating $\boldsymbol{\Sigma}$ with:

$$
\hat{\boldsymbol{\Sigma}}=\left(\begin{array}{c|c}
\hat{A} & \hat{B} \\
\hline \hat{C} & \hat{D}
\end{array}\right) \in \mathbb{R}^{(k+p) \times(k+m)}
$$

where as before $k<n$ (or $k \ll n$ ) and the above four conditions are satisfied.

\section{Motivating Examples}

Models of dynamical systems are useful primarily for two reasons: first for simulation and second for control. We will illustrate these reasons with two examples, namely modeling of VLSI circuits: where the effect of interconnections need to be simulated, and control of the International Space Station (ISS): where low order controllers are necessary.

A. High-frequency, sub-micron VLSI circuits. The scaling trends in today's VLSI circuits are: decrease of feature size (by more than $10 \%$ per year), increase of chip size (by more than $10 \%$ per year); this results in an overall increase in chip complexity (by more than 50\% per year). One consequence of these trends is the multi-layered design (currently a chip may consist of up to 10 layers). Furthermore there is an increase in operating frequency, which has reached the gigahertz range. These developments have had an impact on physical parameters, the most important being the increase of interconnect length. What used to be a piece of wire linking two components on the chip, now needs to be modeled. In current sub-micron designs (feature size in the submicron range), the interconnect length is of the order of several kilometers, the number of transistors approaches one billion and the operating frequency surpasses $1 \mathrm{GHz}$.

In conclusion, before settling on a particular design, it is important to be able to quickly simulate the particular chip layout and check that the signal delays, for instance from an input to a logic gate, does not exceed certain limits. Thus interconnections must be modeled. The resulting models are very high-order: $n \approx 10^{5} \ldots 10^{6}$. The usual simulation tool SPICE, turns out to be inadequate for such complex circuits. For an account of intertconnect issues see [73]. Model reduction was introduced into this area by Rohrer; see [64, 32].

B. ISS: International Space Station. This is a complex structure composed of many modules (it is estimated that more than 40 Shuttle flights will be required to complete the assembly). Furthermore each module is described in terms of $n \approx 10^{3}$ state variables.

In this case controllers will be needed, for instance, to reduce oscillatory motion or to fix the orientation of the space station with respect to some desired direction. It is well known that generically a controller of a given plant has the same dimension as the plant. Since these controllers have to be implemented on-board, they must have low complexity due to hardware, radiation, throughput, testing limitations. Thus reduced order models are very useful for the development of reduced-order controllers.

C. In general: Systems described by PDEs. Approximate finite element (FE) equations are obtained through spatial discretization of certain time dependent systems which are described by PDEs, such as parabolic equations subject to boundary control. These equations are of high complexity. One such case is presented later as an example.

Conclusion. In all these cases, any realistic model will have high complexity, in other words it will require many state variables to be adequately described. The resulting complexity, i.e. number of first-order differential equations, is such that a simplification or model reduction will be needed in order to perform a simulation in an amount of time which is acceptable for the application at hand, or for the design of a low order controller which achieves desired objectives. Thus in all these cases reduced-order models are needed. The issues with large-scale systems are: 


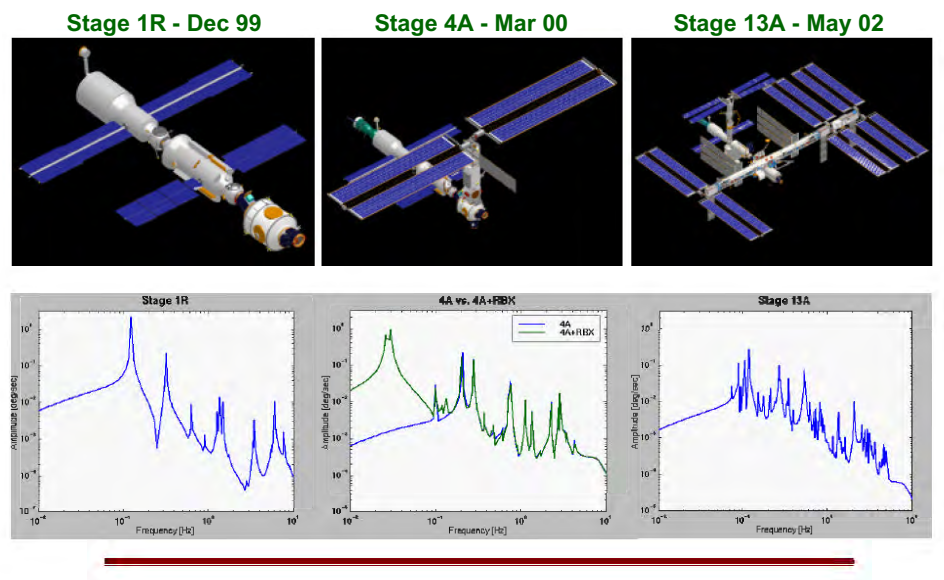

Figure 1: ISS: Evolution of the frequency response as more components are added (Data: Draper Labs).

\section{- Storage.}

\section{- Computational speed.}

- Accuracy: large-scale problems are likely to be ill-conditioned.

- In addition: we need global error bounds and preservation of stability/passivity.

Due to the third item above, what works for dense computations involving low-dimensional systems, may not work in a large-scale setting.

\section{Approximation methods}

Approximation methods can be cast into three broad categories: (a) SVD based methods, (b) Krylov based methods, (c) Iterative methods combining aspects of both the SVD and Krylov methods. We will analyze them in the sequel and point out their strengths and weaknesses.

The SVD-based approximation methods have their roots in the Singular Value Decomposition and the resulting solution of the approximation of matrices by means of matrices of lower rank, which are optimal in the 2-norm (or more generally in unitarily invariant norms). The quantities which are important in deciding to what extent a given finite-dimensional operator can be approximated by one of lower rank are the so-called singular values; these are the square roots of the eigenvalues of the product of the operator and its adjoint. Important is that the ensuing error satisfies an apriori computable upper bound.

The question which arises is whether this result can be applied or extended to the case of dynamical systems. One straightforward way of applying it to a dynamical system described by (1) is as follows. Choose an input function and compute the resulting trajectory; collect samples of this trajectory at different times and compute the SVD of the resulting collection of samples. Then apply the SVD to the resulting matrix. This is a method which is widely used in computation involving PDEs; it is known as Proper Orthogonal Decomposition (POD). The problem however in this case is that the resulting simplification heavily depends on the initial excitation function chosen, the time instances at which the measurements are taken; consequently, the singular values obtained are not system invariants. The advantage of this method is that it can be applied to high-complexity linear as well as nonlinear systems. 
A different approach results in the case of linear systems (2). First we observe that a linear time-invariant dynamical system $\boldsymbol{\Sigma}$, can be represented by means of an integral (convolution) operator; if in addition $\boldsymbol{\Sigma}$ is finite dimensional, then this operator has finite rank and is hence compact. Consequently it has a set of finitely many nonzero singular values. Thus in principle, the SVD approximation method can be used to simplify such dynamical systems.

Indeed, there is a set of invariants called the Hankel singular values which can be attached to every linear, constant, finite-dimensional system. These invariants play the same role for dynamical systems that the singular values play for constant finite-dimensional matrices. In other words, they determine the complexity of the reduced system and at the same time, they provide a global error bound for the resulting approximation. This gives rise to two model reduction methods, namely: balanced model reduction and Hankel norm approximation. Many have observed that the Hankel singular values of many systems decay extremely rapidly. Hence very low rank approximations are possible and accurate low order reduced models will result.

The first decisive results in this direction were obtained by Adamjan, Arov, and Krein [1,2]. The theory as it is used today is due to Glover [36]. Independently, Moore [60], developed the concept of balancing; this led to the very popular method of approximation by balanced truncation.

The limitation of this approach comes from the fact that the computation of the Hankel singular values involves the solution of two linear matrix equations, the Lyapunov equations. Subsequently, the eigenvalues of the product of two positive definite matrices is required; these are the reachability and observability grammians. The exact solution of such equations requires dense computations and therefore can roughly speaking be carried out for systems of modest dimension $n \approx$ a few hundred.

A modification of this method has been developed for nonlinear systems. It is the method of empirical grammians. Its goal is to remedy the issues arising in POD methods, at the expense of added computational complexity [52].

\begin{tabular}{|c|c|c|}
\hline \multicolumn{3}{|c|}{ Approximation methods for dynamical systems } \\
\hline \multicolumn{2}{|c|}{$\circ$ SVD } & ○ Krylov \\
\hline Nonlinear Systems & \multicolumn{2}{|c|}{ Linear Systems } \\
\hline $\begin{array}{l}\text { - POD methods } \\
\text { - Empirical grammians }\end{array}$ & $\begin{array}{l}\text { - Balanced truncation } \\
\text { - Hankel approximation }\end{array}$ & $\begin{array}{l}\text { - Realization } \\
\text { - Interpolation } \\
\text { - Lanczos } \\
\text { - Arnoldi }\end{array}$ \\
\hline \multicolumn{3}{|c|}{ ○ SVD-Krylov } \\
\hline
\end{tabular}

Table 1: Overview of approximation methods

A different set of approximation methods have been developed. They are iterative in nature and hence can be applied to very high order systems. These are the Krylov-based approximation methods, which do not rely on the computation of singular values. Instead they are based on moment matching of the impulse response of $\boldsymbol{\Sigma}$. Two widely used methods fall under this category, namely the Lanczos and the Arnoldi procedures, which were put forward by C. Lanczos in 1950 [54] and by W.E. Arnoldi in 1951 [16], respectively. These methods have been very influential in iterative eigenvalue computations and more recently in model reduction. Their drawbacks are that the resulting reduced order systems have no guaranteed error bound, stability is not necessarily preserved and some of them are not automatic. 
Two leading efforts in this area are Padé via Lanczos (PVL) $[29,19,30]$, and multipoint rational interpolation [39]. The PVL approach exploits the deep connection between the (nonsymmetric) Lanczos process and classical moment matching techniques, i.e. the fundamental connection of Krylov subspace projection methods in linear algebra to the partial realization problem in system theory as laid out in [37, 38]; see also [22, 33, 29, 39, 74, 48]. A closely related approach which has been applied to VLSI circuits can be found in [50]; see also [59].

The multipoint rational interpolation approach utilizes the rational Krylov method of Ruhe [67] to provide moment matching of the transfer function at selected frequencies and hence to obtain enhanced approximation of the transfer function over a broad frequency range. These techniques have proven to be very effective. PVL has enjoyed considerable success in circuit simulation applications. Rational interpolation achieves remarkable approximation of the transfer function with very low order models. Nevertheless, there are shortcomings to both approaches. In particular, since the methods are local in nature, it is difficult to establish global error bounds. Heuristics have been developed that appear to work, but no global results exist. Secondly, the rational interpolation method requires selection of interpolation points. At present, this is not an automated process and relies on ad-hoc specification by the user.

This brings us to the third class of methods which seek to combine the best features of the SVD based methods and of the Krylov based methods. We will refer to these as the SVD-Krylov-based approximation methods. The essential feature of the former is the solution of Lyapunov equations and the essential feature of the latter is the use of Krylov spaces (also known in system theory as reachability-observability subspaces). In particular it uses an iterative computation of projections onto these spaces. This gives rise to various approaches which have iterative Lyapunov solvers at their center.

The first approach in the third category makes use of a recent advance in the computation of eigenvalues of large matrices, called implicit restarting [70]. This has been applied with some success to the construction of stable reduced models to known stable LTI systems [40]. The approach proposed by [48], uses an implicitly restarted dual Arnoldi approach. The dual Arnoldi method runs two separate Arnoldi processes, one for the controllability subspace, and the other for the observability subspace and then constructs an oblique projection from the two orthogonal Arnoldi basis sets. The basis sets and the reduced model are updated using a generalized notion of implicit restarting. The updating process is designed to iteratively improve the approximation properties of the model. Essentially, the reduced model is reduced further, keeping the best features, and then expanded via the dual Arnoldi processes to include new information. The goal is to achieve approximation properties related to balanced realizations.

Other related approaches $[23,59,63]$ work directly with projected forms of the two Lyapunov equations $(5)$ to obtain low rank approximations to the system grammians.

One problem in working with the two Lyapunov equations separately and then applying dense methods to the reduced equations is consistency. One cannot be certain that the two separate basis sets are the ones that would have been selected if the full system grammians had been available. We are not aware of a consistent implicit restarting scheme that would be effective in converging the two separate processes towards the balancing transformation corresponding to the largest Hankel singular values.

This difficulty led to the approach described in [4], and makes use of a third type of grammian, called the cross-grammian. The primary goal of this approach is to develop implicit restarting methods that will iteratively produce approximations of specified rank $k$ to controllability, observability, and cross grammians that are best approximations of rank $k$ to the full grammians. These considerations lead to algorithms within the implicit restarting framework that will iteratively improve a set of $k$ leading singular values and the corresponding $k$ dimensional Krylov basis instead of computing all the singular values and truncating. This reduces computational costs from $\mathcal{O}\left(n^{3}\right)$ to $\mathcal{O}\left(n \cdot k^{2}\right)$, and storage costs from $\mathcal{O}\left(n^{2}\right)$ to $\mathcal{O}(n \cdot k)$.

A second approach along the same lines was developed by Hodel [44]. It proposes an approximate power method for the iterative solution of the Lyapunov equation.

In the sections that follow we will describe the methods mentioned above in more detail. 


\subsection{SVD-based approximation methods}

\subsubsection{The Singular value decomposition: SVD}

Every matrix $A \in \mathbb{R}^{n \times m}$ can be decomposed in a product of 3 matrices [41]:

$$
A=U \Sigma V^{*} \in \mathbb{R}^{n \times m}
$$

where $U$ and $V$ are unitary (orthogonal) and $\Sigma=\operatorname{diag}\left(\sigma_{1}, \cdots, \sigma_{n}\right) \in \mathbb{R}^{n \times m}$, is diagonal with nonnegative diagonal entries called singular values: $\sigma_{i}=\sqrt{\lambda_{i}\left(A^{*} A\right)} \geq \sigma_{i+1}$, and the 2-induced norm of $A$ is $\sigma_{1}$. The left singular vectors are: $U=\left(\begin{array}{llll}u_{1} & u_{2} & \cdots & u_{n}\end{array}\right), U U^{*}=I_{n}$, and the right singular vectors are: $V=\left(\begin{array}{llll}v_{1} & v_{2} & \cdots & v_{m}\end{array}\right), V V^{*}=$ $I_{m}$. Finally, the dyadic decomposition of $A$ is:

$$
A=\sigma_{1} u_{1} v_{1}^{*}+\sigma_{2} u_{2} v_{2}^{*}+\cdots+\sigma_{n} u_{n} v_{n}^{*}
$$

Optimal approximation in the 2-norm. Given: $A \in \mathbb{R}^{n \times m}$, find: $X \in \mathbb{R}^{n \times m}$, rank $X=k<\operatorname{rank} A$, such that an appropriate norm of the error $E=A-X$ is minimized. This problem can be solved explicitly if we take as norm the 2-norm, or any unitarily invariant norm. Although the problem is non-convex, it readily follows that a special decomposition, namely the singular value decomposition (SVD), provides the solution. The theorem that follows is attributed to several people. Two surveys in this regard are worth noting, namely [24] and [47].

Theorem (Schmidt-Mirsky, Eckart-Young). The solution of the above problem is:

$$
\min _{\operatorname{rank} X \leq k}\|A-X\|_{2}=\sigma_{k+1}(A)
$$

A non-unique minimizer $X_{\#}$ is obtained by truncating the dyadic decomposition of $A$ : $X_{\#}=\sigma_{1} u_{1} v_{1}^{*}+\sigma_{2} u_{2} v_{2}^{*}+$ $\cdots+\sigma_{k} u_{k} v_{k}^{*}$.
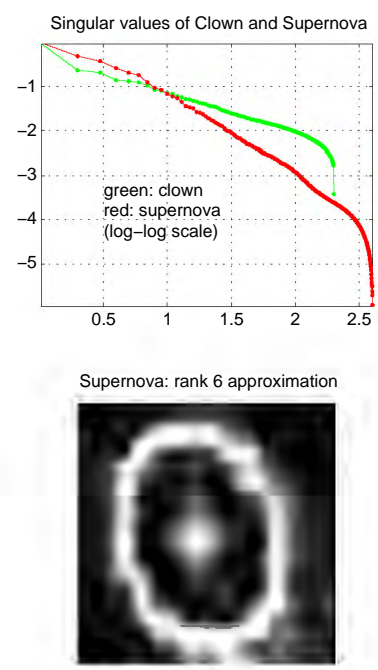
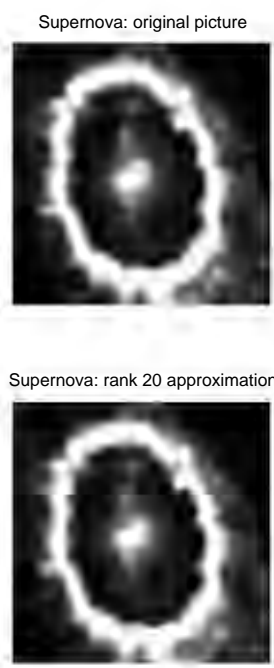
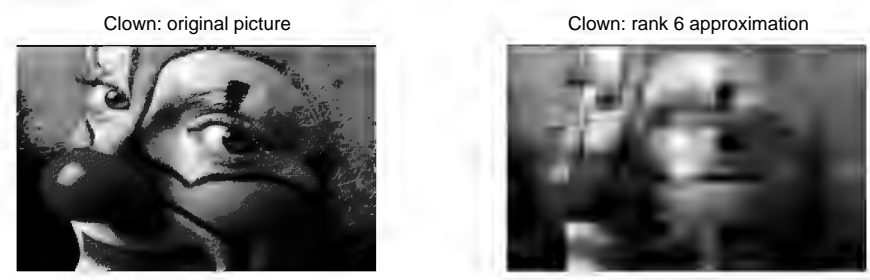

Clown: rank 12 approximation

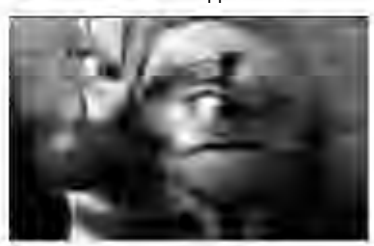

Clown: rank 20 approximation

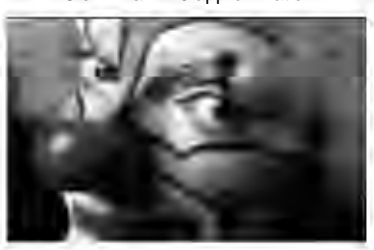

Figure 2: Approximation of the "Supernova" and "Clown" by images of lower rank

Example. In figure 2 a supernova picture and the clown picture from MatLab are considered. They can be represented by means of a $406 \times 406$, and a $200 \times 320$ matrix, respectively, each entry (pixel) having a value between 0 (white) and 1 (black) in 64 levels of gray. Both matrices have full rank, i.e. 404 and 200, respectively. Their 
numerical ranks however are much lower. The singular values of these two images are shown in the top-right-handside subplot on a log-log scale; both sets of singular values fall-off rapidly, and hence low-rank approximations with small error (distortion) are possible. By superimposing the two singular value plots, we can also draw conclusions on the relative error for approximants of the same rank. Thus for approximants of rank up to 10, the clown image is easier to approximate, while for approximants of rank higher than 10, the converse is true. For the left-handside plots, clockwise from top we have: the singular values, the original, and approximants of rank 20 and 6 , respectively. For the right-hand-side plots, clockwise from top: original, and approximants of rank 6, rank 20, and rank 12 , respectively.

\subsubsection{Proper Orthogonal Decomposition (POD) methods}

Consider the nonlinear system given by (1). For a fixed input $u$, the state trajectory at certain instances of time $t_{k}$ is measured:

$$
\mathcal{X}=\left[x\left(t_{1}\right) x\left(t_{2}\right) \cdots x\left(t_{N}\right)\right] \in \mathbb{R}^{n \times N}
$$

This is called a matrix of snapshots of the state. In general $N \gg n$. Next the singular value decomposition of $\mathcal{X}$ is computed. If the singular values of this matrix fall off rapidly, a low-order approximation of this system can be computed.

$$
\mathcal{X}=U \Sigma V^{*} \approx U_{k} \Sigma_{k} V_{k}^{*}, k \ll n
$$

Equivalently, we can compute the eigenvalue decomposition of the grammian

$$
\mathcal{X} \mathcal{X}^{*}=\sum_{t_{i}} x\left(t_{i}\right) x\left(t_{i}\right)^{*}=U \Sigma \Sigma^{*} U^{*} \approx U_{k} \Sigma_{k} \Sigma_{k}^{*} U_{k}^{*}, k \ll n
$$

Let $x(t) \approx U_{k} \xi(t), \xi(t) \in \mathbb{R}^{k}$. Then $U_{k} \dot{\xi}(t)=f\left(U_{k} \xi(t), u(t)\right)$, which implies the reduced order state equation:

$$
\dot{\xi}(t)=U_{k}^{*} f\left(U_{k} \xi(t), u(t)\right), y(t)=h\left(U_{k} \xi(t), u(t)\right)
$$

Thus the approximation $\xi(t)$ of the state $x(t)$ evolves on a low-dimensional space which is spanned by the $k$ leading columns of $U$, that is by the leading left singular vectors of $\mathcal{X}$. For details see [65, 21, 82]. An improved version of this method is given in [52].

\subsubsection{Approximation by balanced truncation}

Consider the system $\boldsymbol{\Sigma}$ given by (3), with $A$ stable. Following the POD method, we consider the output to a particular input, namely, the impulse $u=\delta$; in this case the output is called impulse response and is denoted by $h(t)=C e^{A t} B, t \geq 0$. This can be decomposed into an input-to-state map: $x(t)=e^{A t} B$, and a state-to-output map: $\eta(t)=C e^{A t}$. Thus the input $\delta(t)$ causes the state $x(t)$, while the initial condition $x(0)$ causes the output $y(t)=\eta(t) x(0)$. The the grammians corresponding to $x$ and $\eta$ are:

$$
\mathcal{P}=\sum_{t} x(t) x(t)^{*}=\int_{0}^{\infty} e^{A t} B B^{*} e^{A^{*} t} d t \text { and } \mathcal{Q}=\sum_{t} \eta(t)^{*} \eta(t)=\int_{0}^{\infty} e^{A^{*} t} C^{*} C e^{A t} d t
$$

Under state transformation $\hat{x}=T x$, the two grammians are transformed by congruence: $\hat{\mathcal{P}}=T \mathcal{P} T^{*}, \hat{\mathcal{Q}}=$ $T^{-*} \mathcal{Q} T^{-1}$; thus $\lambda_{i}(\mathcal{P} \mathcal{Q})$ are input-output invariants of $\boldsymbol{\Sigma}$, called the Hankel singular values of $\boldsymbol{\Sigma}$. These are fundamental invariants which determine how well $\boldsymbol{\Sigma}$ can be approximated by a reduced-order $\hat{\boldsymbol{\Sigma}}$. They play the same role for dynamical systems that the singular values play for finite-dimensional matrices. Their computation is therefore of primary importance. 


\subsubsection{Computation of the singular values}

The key for the computation of the Hankel singular values is the observation that the grammians $\mathcal{P}$ and $\mathcal{Q}$ are the unique Hermitian positive definite solutions to the following linear matrix equations, which are known as Lyapunov equations:

$$
A \mathcal{P}+\mathcal{P} A^{*}+B B^{*}=0, \quad A^{*} \mathcal{Q}+\mathcal{Q} A+C C^{*}=0 \Rightarrow \sigma_{i}(\boldsymbol{\Sigma})=\sqrt{\lambda_{i}(\mathcal{P Q})}
$$

then the Hankel singular values $\sigma_{i}(\boldsymbol{\Sigma})$ of the system $\boldsymbol{\Sigma}$ are the square roots of the eigenvalues of the product $\mathcal{P} \mathcal{Q}$.

Numerically stable algorithms to solve these equations are well known (see e.g. [79]). One can directly compute an upper triangular $U$ with $\mathcal{P}=U U^{*}$ and a lower triangular $L$ with $\mathcal{Q}=L L^{*}$. If $A, B, C$ are real then $U$ and $L$ are also real. The Hankel singular values are computed as the singular values of the product $U^{*} L$ and, with care, this may be computed directly without actually forming the product $[20,43,56]$.

Denoting the SVD of $U^{*} L$ by $U^{*} L=Z \Sigma Y^{*}$, a balanced realization may be constructed through a balancing transformation $T_{b}$ defined by

$$
T_{b}=\Sigma^{\frac{1}{2}} Z^{*} U^{-1}=\Sigma^{-\frac{1}{2}} Y^{*} L^{*} \text { and } T_{b}^{-1}=\mathbf{U} Z \Sigma^{-\frac{1}{2}}=L^{-*} Y \Sigma^{\frac{1}{2}}
$$

Thus in order to avoid inverting possibly not so well conditioned matrices the formulas for $T_{b}$ and $T_{b}^{-1}$ involving only the inverse of $\Sigma$ are used. Under this transformation, the solutions to the two Lyapunov equations become equal and diagonal: $\mathcal{P}=\mathcal{Q}=\Sigma=\operatorname{diag}\left(\sigma_{1}, \cdots, \sigma_{n}\right)$, and

$$
A_{b} \Sigma+\Sigma A_{b}^{*}+B_{b} B_{b}^{*}=0, A_{b}^{*} \Sigma+\Sigma A_{b}+C_{b}^{*} C_{b}=0,
$$

where $A_{b}=T_{b} A T_{b}^{-1}, B_{b}=T_{b} B, C_{b}=C T_{b}^{-1}$. Model reduction is now possible through simple truncation. Let $\Sigma_{2}$ contains the small Hankel singular values and partition (in the balanced basis):

$$
A_{b}=\left(\begin{array}{l|l}
A_{11} & A_{12} \\
\hline A_{21} & A_{22}
\end{array}\right), B=\left(\begin{array}{c}
B_{1} \\
\hline B_{2}
\end{array}\right), C=\left(C_{1} \mid C_{2}\right), \Sigma=\left(\begin{array}{c|c}
\Sigma_{1} & 0 \\
\hline 0 & \Sigma_{2}
\end{array}\right)
$$

The reduced order model is obtained by simple truncation $\hat{\Sigma}=\left(\begin{array}{c|c}A_{11} & B_{1} \\ \hline C_{1} & \end{array}\right)$, that is, by taking the $k \times k$, $k \times m, p \times k$ leading blocks of $A_{b}, B_{b}, C_{b}$, respectively; this system satisfies a $k$-th order Lyapunov equations with diagonal solution $\Sigma_{1}$. This truncation leads to a balanced reduced order system. If this truncation is such that the resulting grammians contain the largest $k$ singular values $\sigma_{1}$ through $\sigma_{k}$, then the $\mathcal{H}_{\infty}$-norm of the error system has the following upper (and lower) bound [36, 27]:

$$
\sigma_{k} \leq\|\boldsymbol{\Sigma}-\hat{\boldsymbol{\Sigma}}\|_{\infty} \leq 2\left(\sigma_{k+1}+\sigma_{k+2}+\ldots+\sigma_{n}\right)
$$

The $\mathcal{H}_{\infty}$ norm $\|\boldsymbol{\Sigma}\|_{\infty}$, of a system $\boldsymbol{\Sigma}$ is defined as the maximum of the highest peak of the frequency response, that is as the largest singular value of the transfer function evaluated on the imaginary axis (i.e. of the frequency response) $\sigma_{\max }\left[D+C(j \omega-A)^{-1} B\right]$. This result provides the rigorous justification for using the reduced order model to predict behavior and enact control of the full system. The upper bound for the error system given above, translates to an upper bound for the energy of the output error signal.

\subsubsection{Other types of balancing}

Besides the balancing based on Lyapunov equations, there are others. The principle is to find a transformation which yields solution to appropriate Lyapunov and/or Riccati equations equal and diagonal [61]. Here is a short description of each one of them. 
- Lyapunov balancing. Solution to Lyapunov equations equal and diagonal: $A \mathcal{P}+\mathcal{P} A^{*}+B B^{*}=0, A^{*} \mathcal{Q}+$ $\mathcal{Q} A+C^{*} C=0$. Reduced model: preserves stability; there exists an error bound.

- LQG balancing. Solution to Riccati equations equal and diagonal: $A \mathcal{P}+\mathcal{P} A^{*}+B B^{*}-\mathcal{P} C^{*} C \mathcal{P}=0$, $A^{*} \mathcal{Q}+\mathcal{Q} A^{*}+C^{*} C-\mathcal{Q} B B^{*} \mathcal{Q}=0$. It can be applied to unstable systems. Reduced system: leads to feedback design for full system.

- Stochastic balancing. Solution to Lyapunov-Riccati equations equal and diagonal: $A \mathcal{P}+\mathcal{P} A^{*}+B B^{*}=0$, $A^{*} \mathcal{Q}+\mathcal{Q} A+C_{W}^{*} C_{W}=0$, where $C_{W}=D^{-1}\left(C-B_{W}^{*} \mathcal{Q}\right)$ and $B_{W}=\mathcal{P} C^{*}+B D^{*}$. This is leads to model reduction which belongs to those satisfying a relative error bound. Truncation preserves the minimum phase property; if $\sigma_{i}$ denote the singular values obtained in this case, the relative error bound is

$$
\left\|H^{-1}\left(H-H_{k}\right)\right\|_{\infty} \leq \prod_{i=k+1}^{n}\left[\frac{1+\sigma_{i}}{1-\sigma_{i}}\right]-1
$$

- Positive real balancing. The (smallest) positive definite solution to two Riccati equations below, equal and diagonal: $A^{*} \mathcal{Q}+\mathcal{Q} A+\left(C^{*}-\mathcal{Q} B\right)\left(D+D^{*}\right)^{-1}\left(C-B^{*} \mathcal{Q}\right)=0, A \mathcal{P}+\mathcal{P} A^{*}+\left(\mathcal{P} C^{*}-B\right)\left(D+D^{*}\right)^{-1}\left(C \mathcal{P}-B^{*}\right)=$ 0. Passivity: $\sigma_{i}<1$. Reduced model: preserves passivity.

\subsubsection{Hankel norm approximation}

Similar method: Hankel norm approximation. This is a direct generalization of the Schmidt-Mirsky, EckartYoung result to integral operators resulting from LTI (linear, time-invariant) systems. We will briefly outline the construction method next. For details we refer to $[1,2,36]$; see also [8, 9] as well as the books $[13,62]$.

\section{Construction of Hankel-norm approximants:}

(1) find $\tilde{\boldsymbol{\Sigma}}$ such that $\boldsymbol{\Sigma}-\tilde{\boldsymbol{\Sigma}}$ is all-pass ${ }^{1}$ with norm $\sigma_{k}$. (2) project $\tilde{\boldsymbol{\Sigma}}$ onto its stable part $\tilde{\boldsymbol{\Sigma}}_{+}$; the optimal approximant is $\hat{\boldsymbol{\Sigma}}=\tilde{\boldsymbol{\Sigma}}_{+}$.

As in the balanced truncation case, Hankel norm approximation has the following guaranteed properties: (1) stability is preserved, and (2) the global error bound (6) holds. Its drawbacks are (1) dense computations, matrix factorizations/inversions are required; these properties imply that the computations may be ill-conditioned. (2) The whole transformed system is needed in order to truncate the de-

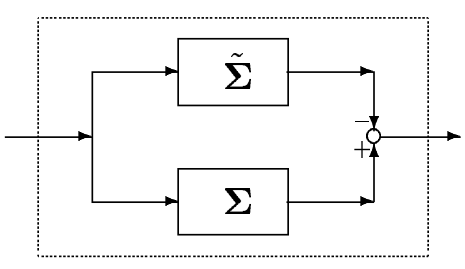
sired subsystem. This leads to a number of operations of the order $\mathcal{O}\left(n^{3}\right)$ and storage of the order $\mathcal{O}\left(n^{2}\right)$.

We conclude this subsection with a series of remarks concerning balanced and Hankel norm approximation.

Remark 3.1 (a) To enhance accuracy, one may obtain the balanced reduced order model without explicitly computing the balancing transformation; this was first introduced in [76].

(b) Due to the importance of the Lyapunov or more generally of the Sylvester equation, in system theory, many authors have studied various aspects of the solution of this equation in detail. For some sample papers we refer besides the classical paper of Lancaster [53], to more recent work of Laub, Simoncini, Avrachenkov [35, 69, 17]; the Sylevster and Lyapunov equations have also been studied in the following papers [25, 26, 35, 44, 45, 46, 63, 68].

(c) Ill-conditioning in balanced truncation. In [66] the following equivalence is developed:

$$
A X+X A^{*}+Q=0 \Leftrightarrow\left(\begin{array}{cc}
A & Q \\
0 & -A^{*}
\end{array}\right)\left(\begin{array}{cc}
I & X \\
0 & I
\end{array}\right)=\left(\begin{array}{cc}
I & X \\
0 & I
\end{array}\right)\left(\begin{array}{cc}
A & 0 \\
0 & -A^{*}
\end{array}\right)
$$

\footnotetext{
${ }^{1}$ A system $\boldsymbol{\Sigma}$ is all-pass or unitary if for all input/output pairs $u, y$ the ratio $\frac{\|y\|}{\|u\|}$ is constant.
} 
Thus with $V_{1}^{*} V_{1}+V_{2}^{*} V_{2}=I$, $\operatorname{det} V_{2} \neq 0, H=-V_{2}^{-1} A^{*} V_{2}$ and

$$
\left(\begin{array}{cc}
A & Q \\
0 & -A^{*}
\end{array}\right)\left(\begin{array}{l}
V_{1} \\
V_{2}
\end{array}\right)=\left(\begin{array}{c}
V_{1} \\
V_{2}
\end{array}\right) H \Rightarrow X=V_{1} V_{2}^{-1}
$$

This means the solution can be computed by computing the eigenspace of the composite matrix belonging to $A$. This requires the decoupling of the spectra of $A$ and $-A^{*}$. The conclusion is that ill-conditioning occurs when $A$ has eigenvalues close to the imaginary axis, i.e. $\boldsymbol{\Sigma}$ has dominant poles close to the imaginary axis.

(d) Canonical form. There is a canonical form associated with balanced representations. In the single-input, single-output (SISO) case, with distinct singular values, the three matrices have the form: $A_{i, j}=-\frac{b_{i} b_{j}}{s_{i} s_{j} \sigma_{i}-\sigma_{j}}$, $B_{i, 1}=b_{i}, C_{1, i}=s_{i} b_{i}$, where $s_{i}$ are $\pm 1, b_{i}>0$ and $\sigma_{i}$ are the Hankel singular values. For details see [61].

(e) Interpretation of balanced basis. Given the state $x$, let $\mathcal{E}_{r}$ denote the minimal amount of input energy needed to steer $\quad 0 \rightarrow x$. Let also, $\mathcal{E}_{o}$ be the maximal output observation energy obtained by observing the output caused by the initial condition $x: x \rightarrow 0$. It follows that $\mathcal{E}_{o}^{2}=x^{*} \mathcal{Q} x, \mathcal{E}_{r}^{2}=x^{*} \mathcal{P}^{-1} x$, and in a balanced basis the product is equal to one: $\mathcal{E}_{r} \cdot \mathcal{E}_{o}=1$.

(f) As already mentioned, balancing for linear systems is equivalent to POD on the impulse response.

(g) Model reduction of unstable or descriptor systems can be carried out by various means, e.g., LQG balancing, frequency domain grammians [83], or normalized coprime factorizations [78].

(h) Weighted approximation is also possible: for instance, frequency weighted balanced truncation with explicit $\mathcal{L}_{\infty}$ error bounds, or weighted Hankel approximation with anti-stable weighting [27, 51, 81].

(i) We conclude these remarks with a comment on the tightness of the bound (6). In fact equality holds for systems $\boldsymbol{\Sigma}$ having zeros which interlace the poles; an example of such systems are electric circuits which are composed of resistors and capacitors (RC circuits).

\subsection{Krylov-based approximation methods}

The moments associated with the linear system $\boldsymbol{\Sigma}(3)$, at some point $s_{0} \in \mathbb{C}$, are defined as the moments of its impulse response $h$ weighted by the exponential $e^{s_{0} t}$, namely

$$
\eta_{k}=\int_{0}^{\infty}(-t)^{k} h(t) e^{-s_{0} t} d t=\left.\frac{d^{k}}{d s^{k}} H(s)\right|_{s=s_{0}}
$$

These numbers can be expressed in terms of the series expansion of the transfer function $H(s)=D+C(s I-$ $A)^{-1} B$ of $\boldsymbol{\Sigma}$ around $s_{0} \in \mathbb{C}$; the transfer function is the Laplace transform of the impulse response:

$$
H(s)=\eta_{0}+\eta_{1}\left(s-s_{0}\right)+\eta_{2}\left(s-s_{0}\right)^{2}+\eta_{3}\left(s-s_{0}\right)^{3}+\cdots
$$

The problem now consists in finding $\hat{\boldsymbol{\Sigma}}(4)$, with

$$
\hat{H}(s)=\hat{\eta}_{0}+\hat{\eta}_{1}\left(s-s_{0}\right)+\hat{\eta}_{2}\left(s-s_{0}\right)^{2}+\hat{\eta}_{3}\left(s-s_{0}\right)^{3}+\cdots
$$

such that for appropriate $\ell$ :

$$
\eta_{j}=\hat{\eta}_{j}, j=1,2, \cdots, \ell
$$

The key to the success of these methods is that they can be implemented iteratively. These implementation methods are closely related to iterative eigenvalue computations [72]. There is thus a strong interaction between model reduction for large-scale systems and large-scale eigenvalue computations; this is explored in [13].

If the expansion is around infinity, the moments are known as Markov parameters, and the problem is known as partial realization; its solution is computed through the Lanczos and Arnoldi procedures. If the expansion is around zero the problem becomes Padé approximation. In general, for arbitrary $s_{0} \in \mathbb{C}$, we have the problem 
of rational interpolation, which amounts to matching moments at the specified point $s_{0}$. This interpolation is obtained indirectly using the so-called rational Lanczos procedure which operates with $\left(A-s_{0} I\right)^{-1}$ in place of $A$. The inverse operation is applied by solving triangular systems obtained from an initial LU decomposition (using a sparse direct method if $A$ is sparse).

It should be noted that the computation of moments is numerically problematic, because the powers of eigenvalues of $A$ grow exponentially fast in general (the eigenvalues of $A$ have negative real parts, many of them bigger in magnitude that one). Thus the key fact for numerical reliability is that if $\boldsymbol{\Sigma}$ is given by means of $(A, B, C)$, Krylov methods allow moment matching without the explicit computation of moments. For an overview as well as details on the material presented in this section we refer to $[37,38,67,42,39,72,74,33,34,7]$.

\subsubsection{The Lanczos and Arnoldi processes}

In this section we will briefly review the Lanczos and Arnoldi processes. For simplicity we will do so in the SISO (single-input single-output) case, i.e. no block versions will be considered. There are two ways of looking at these algorithms: the conventional one and the system-theoretic one, which is less common.

Conventional representation. Given $A \in \mathbb{R}^{n \times n}$, and vectors $B, C^{*} \in \mathbb{R}^{n}$, the two-sided Lanczos and the Arnoldi processes construct at each step matrices $W_{k}, V_{k} \in \mathbb{R}^{n \times k}$, such that the following relationships are satisfied:

\begin{tabular}{|c|c|}
\hline \multicolumn{2}{|c|}{ Lanczos and Arnoldi algorithms } \\
\hline Lanczos & Arnoldi \\
\hline \multicolumn{2}{|c|}{ Initialization } \\
\hline$V_{1}=\frac{1}{\sqrt{|C B|}} B, W_{1}=\frac{\operatorname{sign}(C B)}{\sqrt{|C B|}} C^{*}$ & $V_{1}=\frac{1}{\|B\|} B$ \\
\hline \multicolumn{2}{|c|}{$t$-th step } \\
\hline $\begin{array}{l}A V_{t}=V_{t} T_{t}+r_{t} e_{t}^{*} \\
A^{*} W_{t}=W_{t} T_{t}^{*}+q_{t} e_{t}^{*} \\
W_{*}^{*} V_{t}=I_{t}, t=2,3, \cdots,\end{array}$ & $\begin{array}{l}A V_{t}=V_{t} H_{t}+g_{t} e_{t}^{*} \\
V_{t}^{*} V_{t}=I_{t}, t=2,3, \cdots,\end{array}$ \\
\hline
\end{tabular}

where $e_{t} \in \mathbb{R}^{n}$ is the $t^{\text {th }}$ unit vector. The columns of $V_{t}$ in the Arnoldi case are orthogonal, while in the Lanczos case the columns of $V_{t}$ and $W_{t}$ are called bi-orthogonal. Furthermore, in the Lanczos case, $T_{t}$ is tridiagonal and in the Arnoldi case $H_{t}$ is upper Hessenberg.

System theoretic representation. We will first define the $t \times n$ observability matrix $\mathcal{O}_{t}$, the $n \times t$ reachability matrix $\mathcal{R}_{t}$, and the $t \times t$ Hankel matrix $\mathcal{H}_{t}$ associated with $\boldsymbol{\Sigma}$ :

$$
\mathcal{O}_{t}=\left[\begin{array}{l}
C \\
C A \\
\vdots \\
C A^{t-1}
\end{array}\right], \mathcal{R}_{t}=\left[\begin{array}{llll}
B & A B & \cdots & A^{t-1} B
\end{array}\right], \mathcal{H}_{t}:=\left[\begin{array}{cccc}
\eta_{1} & \eta_{2} & \cdots & \eta_{t} \\
\eta_{2} & \eta_{3} & \cdots & \eta_{t+1} \\
\vdots & & \ddots & \\
\eta_{t} & \eta_{t+1} & \cdots & \eta_{2 t-1}
\end{array}\right]
$$

It is well known that $\mathcal{H}_{t}=\mathcal{O}_{t} \mathcal{R}_{t}$. The key step in the Lanczos procedure is to compute the $\mathbf{L} \mathbf{U}$ factorization of $\mathcal{H}_{k}$ :

$$
\mathcal{H}_{t}=L U=\mathcal{O}_{t} \mathcal{R}_{t}
$$

where $L$ lower triangular and $U$ upper triangular, with $L(i, i)= \pm U(i, i)$. Such a factorization exists provided that $\operatorname{det} \mathcal{H}_{t} \neq 0$, for all $t$. We now define the projection $\pi_{R} \pi_{L}$, where:

$$
\pi_{L}=L^{-1} \mathcal{O}_{t} \text { and } \pi_{R}=\mathcal{R}_{t} U^{-1}
$$


Similarly, the key step in the Arnoldi algorithm is the computation of the QR factorization of $\mathcal{R}_{t}$ :

$$
\mathcal{R}_{t}=V U
$$

where $V \in \mathbb{R}^{n \times t}, V^{*} V=I_{t}$, and $U$ is upper triangular; this yields the projection $\pi_{R} \pi_{L}$, where $\pi_{R}=\pi_{L}^{*}=V$. These two projections are used to define reduced order systems $\hat{\boldsymbol{\Sigma}}$ :

$$
\hat{\boldsymbol{\Sigma}}=\left(\begin{array}{c|c}
\hat{A} & \hat{B} \\
\hline \hat{C} &
\end{array}\right), \hat{A}:=\pi_{L} A \pi_{R}, \hat{B}=\pi_{L} B, \hat{C}=C \pi_{R}
$$

Properties: (a) $\pi_{L} \pi_{R}=1$, and (b) $\pi_{R} \pi_{L}$ is a projection. In the Lanczos case the reduced system let the number of moments matched be $2 k$; then: (i) $\hat{\Sigma}$ matches $2 k$ Markov parameters; (ii) $\hat{A}$ is tridiagonal; (iii) $\hat{B}, \hat{C}^{*}$ are multiples of the unit vector $e_{1}$. In the Arnoldi case let the number of moments matched be $k$; the reduced system (i) matches

\begin{tabular}{|c|c|}
\hline \multicolumn{2}{|c|}{ Lanczos/Arnoldi: Iterative Implementation } \\
\hline $\begin{array}{l}\text { 1. } \beta_{1}:=\sqrt{\left|B^{*} C^{*}\right|}, \gamma_{1}:=\operatorname{sgn}\left(B^{*} C^{*}\right) \beta_{1}, \\
v_{1}:=B / \beta_{1}, w_{1}:=C^{*} / \gamma_{1} \\
\text { 2. For } j=1, \cdots, k \text {, set } \\
\qquad \begin{array}{l}\text { (a) } \alpha_{j}:=w_{j}^{*} A v_{j} \\
\text { (b) } r_{j}:=A v_{j}-\alpha_{j} v_{j}-\gamma_{j} v_{j-1}, \\
\\
q_{j}:=A^{*} w_{j}-\alpha_{j} w_{j}-\beta_{j} w_{j-1} \\
\text { (c) } \beta_{j+1}=\sqrt{\left|r_{j}^{*} q_{j}\right|}, \gamma_{j+1}=\operatorname{sgn}\left(r_{j}^{*} q_{j}\right) \beta_{j+1} \\
\text { (d) } v_{j+1}=r_{j} / \beta_{j+1}, w_{j+1}=q_{j} / \gamma_{j+1}\end{array}\end{array}$ & 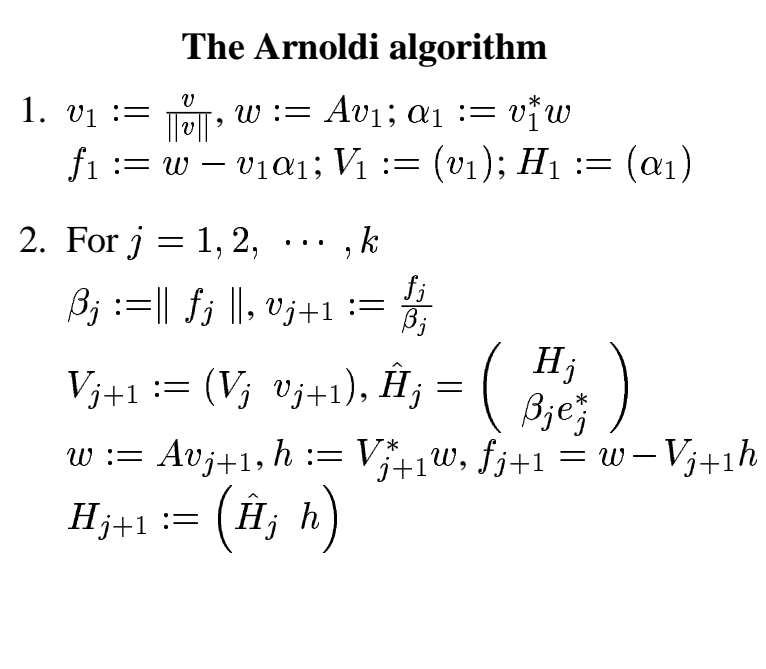 \\
\hline
\end{tabular}
$k$ Markov parameters, (ii) $\hat{A}$ is in upper Hessenberg form, and (iii) $\hat{B}$ is a multiple of $e_{1}$.

The results quoted above are based on the following lemma.

\section{Lemma 3.1 Degree Lemma.}

Arnoldi process. Consider $V \in \mathbb{R}^{n \times k} H \in \mathbb{R}^{k \times k}$, which are generatedat the $k$-th step of the Arnoldi process. For any polynomial $\phi$ of degree less than or equal to $k$, there holds:

$$
\phi(A) V e_{1}=V \phi(H) e_{1}, \operatorname{deg} \phi<k ; \phi(A) V e_{1}=V \phi(H) e_{1}+f \gamma, \operatorname{deg} \phi=k, \gamma \in \mathbb{R}
$$

Lanczos process. Consider $V, W \in \mathbb{R}^{n \times k}, T \in \mathbb{R}^{k \times k}$, which are generated at the $k$-th step by the Lanczos process. For any polynomial $\phi$ of degree less than or equal to $k$, there hold:

$$
\begin{array}{ccc}
\hline \phi(A) V e_{1}=V \phi(T) e_{1} ; & \phi\left(A^{*}\right) W e_{1}=W \phi\left(T^{*}\right) e_{1} & \operatorname{deg} \phi<k \\
\hline \phi(A) V e_{1}=V \phi(T) e_{1}+r \gamma ; & \phi\left(A^{*}\right) W e_{1}=W \phi\left(T^{*}\right) e_{1}+q \eta & \operatorname{deg} \phi=k, \gamma, \eta \in \mathbb{R}
\end{array}
$$


From this simple property it follows that

$$
\begin{aligned}
& c A^{j} b=c A^{j} v_{1}=c V_{k} H_{k}^{j} e_{1}=e_{1}^{*} H_{k}^{j} e_{1}, 1 \leq j<k \text { for Arnoldi } \\
& c A^{\ell+j} b=w_{1}^{*} A^{\ell} A^{j} v_{1}=\left(e_{1}^{*} T_{k}^{\ell} W_{k}^{*}\right)\left(V_{k} T_{k}^{j} e_{1}\right)=e_{1}^{*} T_{k}^{\ell+j} e_{1}, 0 \leq \ell \leq k-1,0 \leq j \leq k \text { for Lanczos }
\end{aligned}
$$

Thus, Arnoldi matches $k$ moments while Lanczos matches $2 k$ moments. In the following table we summarize the three different moment matching methods, together with the moments matched, the points in the complex plane

\begin{tabular}{|c|c|c|c|}
\hline \multicolumn{4}{|c|}{ Krylov methods } \\
\hline Method & "Moments matched & Points in $\mathbb{C}$ & Number of moments \\
\hline 1. $k$ steps Arnoldi/Lanczos & $c A^{j} b$ & $\infty$ & $k / 2 k$ \\
\hline Shift-Invert & $c\left(A-\sigma_{0} I\right)^{-j-1} b$ & $\sigma_{0}$ & $k / 2 k$ \\
\hline Rational Krylov & $c\left(A-\sigma_{i} I\right)^{-j-1} b$ & $\sigma_{1}, \cdots, \sigma_{i}$ & $k=\ell_{1}+\cdots+\ell_{i}$ \\
\hline
\end{tabular}
where they are matched, and the number of moments matched.

Implicitly restarted Arnoldi and Lanczos procedures. The issue here is to get a better approximation of some desired set of preferred eigenvalues, for example, those eigenvalues that have, for instance largest modulus or largest/smallest positive/negative real part. An idea due to Sorensen [70, 57] is to accomplish this by projecting onto the space of desired features, by restarting Lanczos and Arnoldi, while staying in the Lanczos/Arnoldi framework. An overview of applications of implicit restarting can be found in [71].

Remark 3.2 (a) In the Arnoldi algorithm, if $A$ is symmetric, then $H_{j}$ is tridiagonal, and the Arnoldi algorithm coincides with the one-sided or symmetric Lanczos algorithm.

(b) The realization and partial realization problems were introduced by Kalman [49]. A generalization thereof is rational interpolation. A general version of this problem has been solved in [14], and with stability constraint in [15].The recursive interpolation problem is studied in [3,6]. For connections of these results with Lanczos, see [7]. A generalization of the tridiagonal form of $A$ for MIMO systems, is described in [5]. The connection between partial realizations and the Lanczos algorithm was studied in [37, 38].

(c) In (numerical) linear algebra the terminology Krylov subspace $\mathcal{K}_{t}$ is used to denote the space spanned by the first $t$ columns of the reachability (or the observability) matrices $\mathcal{K}_{t}=\operatorname{span} \operatorname{col} \mathcal{R}_{t}$. In system theory these subspaces are known are reachability, observability subspaces, respectively.

(c) The residual in the Arnoldi case is $f_{j}=A v_{j}-V_{j} h_{j}$, where $h_{j}$ is chosen so that the norm $\left\|f_{j}\right\|$ is minimized. It turns out that $V_{j}^{*} h_{j}=0$, and $h_{j}=V_{j}^{*} A v_{j}$, that is $f_{j}=\left(I-V_{j} V_{j}^{*}\right) A v_{j}$.

(d) Comparison between Lanczos and Arnoldi. These two algorithms are similar but have important differences. While Lanczos yields a Padé type approximation, this is not true for Arnoldi. Then, Arnoldi is numerically stable, while Lanczos is not. The number of operations needed to compute a $k$-th order approximant is $\mathcal{O}\left(k^{2} n\right)$ for both (as opposed to $\mathcal{O}\left(n^{3}\right)$ operations required for SVD-based methods). The storage required is $2 k n$ in both cases. The basic operation is $w=A v$, and $\hat{v}=A v, \hat{w}=A^{*} w$, respectively, and the cost is $2 k$; this is followed by $v_{+}=\left(I-V V^{*}\right) w$, and $v_{+}, w_{+}$which have a cost of $n \mathcal{O}\left(k^{2}\right)$. Finally, it should be mentioned that the Lanczos process satisfies a 3-term recurrence relation, while Arnoldi does not; this implies the definition of orthogonal polynomials associated with Lanczos.

(e) The implementation of Krylov methods require matrix-vector multiplications exclusively, no matrix factorizations and/or inversions are needed. Furthermore, there is no need to compute the transformed $n$-th order model and then truncate. This means the absence of ill-conditioning

(f) Drawbacks. 
- there is no global error bound; for a local result see [18].

- $\hat{\boldsymbol{\Sigma}}$ may not be stable, even if $\boldsymbol{\Sigma}$ is stable. Remedy: implicit restart [40].

- Lanczos breaks down if det $\mathcal{H}_{i}=0$. Remedy: look-ahead methods [42].

- $\hat{\boldsymbol{\Sigma}}$ tends to approximate the high frequency poles of $\boldsymbol{\Sigma}$. Hence the steady-state error may be significant. Remedy: match expansions around other frequencies (rational Lanczos) [67, 34, 39].

\subsection{SVD-Krylov-based approximation methods}

Up to this point we briefly reviewed the merits and difficulties associated with two fundamental ways of carrying out model reduction. We reached the conclusion that $S V D$-based approximation methods have a number of desirable properties, namely, there exists a global error bound and stability is preserved. The drawback however is that applicability is restricted to relatively low dimensions. On the other hand, the Krylov-based methods, remedied the latter issue (i.e. applicability) but had a number of important drawbacks, namely, no guaranteed stability preservation and no global error bound. These considerations give rise to the SVD-Krylov-based approach, which aims at combining the best attributes of the two previously described approximation methods.

The basic ingredients of SVD-based methods which gives rise to all the nice properties of this approach, are the two Lyapunov equations used to compute the grammians. On the other hand, the basic ingredients of the Krylov-based methods are precisely the Krylov subspaces, i.e. the span of the columns of the reachability and observability matrices, and the iterative way of computing projections onto these subspaces. The SVD-Krylov methods, thus aim at combining these two sets of attributes by seeking iterative solutions of Lyapunov or more generally of Sylvester equations. In so doing the hope is that a method will be devised which combines the best attributes of the SVD and Krylov methods. Such a method does not exist yet. The quest however continues.

Two methods in this category are $[4,48,44]$. In the sequel we will briefly describe the approach in the former reference.

The difficulty (pointed out earlier) of working with two Lyapunov equations separately, as proposed in [48], has led to the consideration of a different approach which is based upon the notion of a cross grammian that was introduced in [28]. The cross grammian $X$ is defined for square systems $(m=p)$ as the solution to the Sylvester equation $A X+X A+B C=0$. In the SISO (single-input single-output) case, the solution $X$ is similar to a Hermitian matrix and the eigenvalues of $X$ are in fact signed singular values of $\boldsymbol{\Sigma}$. In fact, it is possible to show in this case that $X^{2}=\mathcal{P} \mathcal{Q}$. Our approach consists essentially in constructing low rank $k$ approximate solutions to this system by setting $X=V \hat{X} W^{*}$ with $W^{*} V=I_{k}$ and projecting using $W, V$ as the reduced basis. This uses an implicit restart mechanism that allows the computation of the best rank $k$ approximation to $X$. This solution leads to an error estimate.

The primary goal of [4] is to develop implicit restarting methods that will iteratively produce approximations of specified rank $k$ to controllability, observability, and cross grammians that are best approximations of rank $k$ to the full grammians. We observe that the Hankel singular values of such systems decay extremely rapidly. Hence very low rank approximations to the system grammians are possible and accurate low order reduced models will result. The approach consists in investigating a non-Krylov method related to the recently developed Jacobi-Davidson method to the large scale eigenvalue problem. This has the advantage of admitting a wider variety of restarting options. However, the advantage is gained at the expense of giving up the Krylov properties which provide deep connections with the fundamental system theory. Nevertheless, our method will be based on a quantity called the cross-grammian and because of this some important system theoretic properties are indeed retained which are not necessarily related to moment matching.

This method uses subspace projection techniques combined with variants of implicit restarting to compute a partially balanced realization directly. Instead of computing all the Hankel singular values and then casting out the smallest ones, an implicit restarting mechanism is used to compute just the largest ones and thereby greatly reduce the computational and storage costs required to produce a $k$-th order reduced model. Thus computational costs are reduced from $\mathcal{O}\left(n^{3}\right)$ to $\mathcal{O}\left(n \cdot k^{2}\right)$, and storage costs from $\mathcal{O}\left(n^{2}\right)$ to $\mathcal{O}(n \cdot k)$. 
We conclude this section by summarizing the salient features of each one of the three methods, in the table below. The features of the third column form a wish list, as there is currently no method which fulfills all of them.

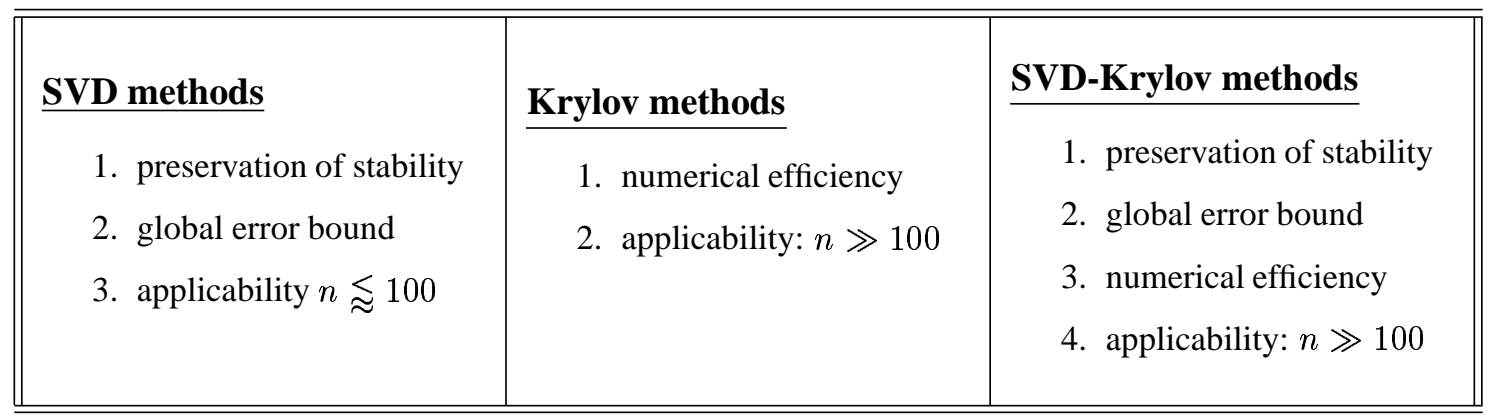

Remark 3.3 - An approximation method which has been popular is model reduction by modal approximation [77]. This method works well when the dominant eigenfrequencies of the original system are of interest and have to be preserved.

- All systems which can be approximated well using SVD-based methods have Hankel singular values which drop off rapidly. Therefore an important issue in model reduction is to determine the rate of decrease of the singular values. Few results in this direction are known, and this remains an open problem [10].

- We would like to draw the readers' attention to two new books, one which already appeared [62], and one which is in preparation $[12,13]$.

- SVD-based model reduction has been extended to time-varying systems; for an overview see [80], [75].

\section{Numerical experiments}

Matlab codes implementing all of the methods mentioned above have already been developed; furthermore a new software library called SLICOT has been developed for control and contains a package which implements all the dense model reduction methods; for details we refer to [79]. A package which is used for large-scale eigenvalue computation is ARPACK [57]. It should be mentioned that one of the largest examples computed involves the determination of stability of a matrix resulting from the discretization of a 3D Navier Stokes equation. The size is several million [58]. This is relevant to the paper at hand because the techniques used are the same as those outlined above.

Most of the problems we have seen exhibit the behavior that is indicative of the success for this approach. Namely, the singular values of $\boldsymbol{\Sigma}$ decay very rapidly indicating that $\boldsymbol{\Sigma}$ is typically approximated well with a reduced order system of very low dimension. In the following example we show the results of computing $\hat{\boldsymbol{\Sigma}}$, where its dimension $k$ is determined by a cut off. We determine $k$ by taking the first $k$ such that

$$
\sigma_{k+1}<\tau \sigma_{1}
$$

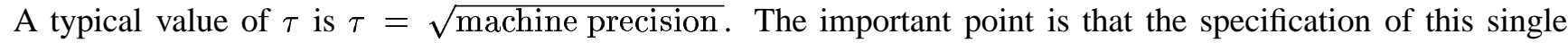
tolerance determines everything in the SVD-based methods. The order of the reduced model and the eigenvalue placement are completely automatic. Moreover, the value of $\tau$ directly determines the error of approximation for $\left\|y-y_{k}\right\|$ over the entire frequency range. As already noted, the key parameters for model reduction are the Hankel singular values. 

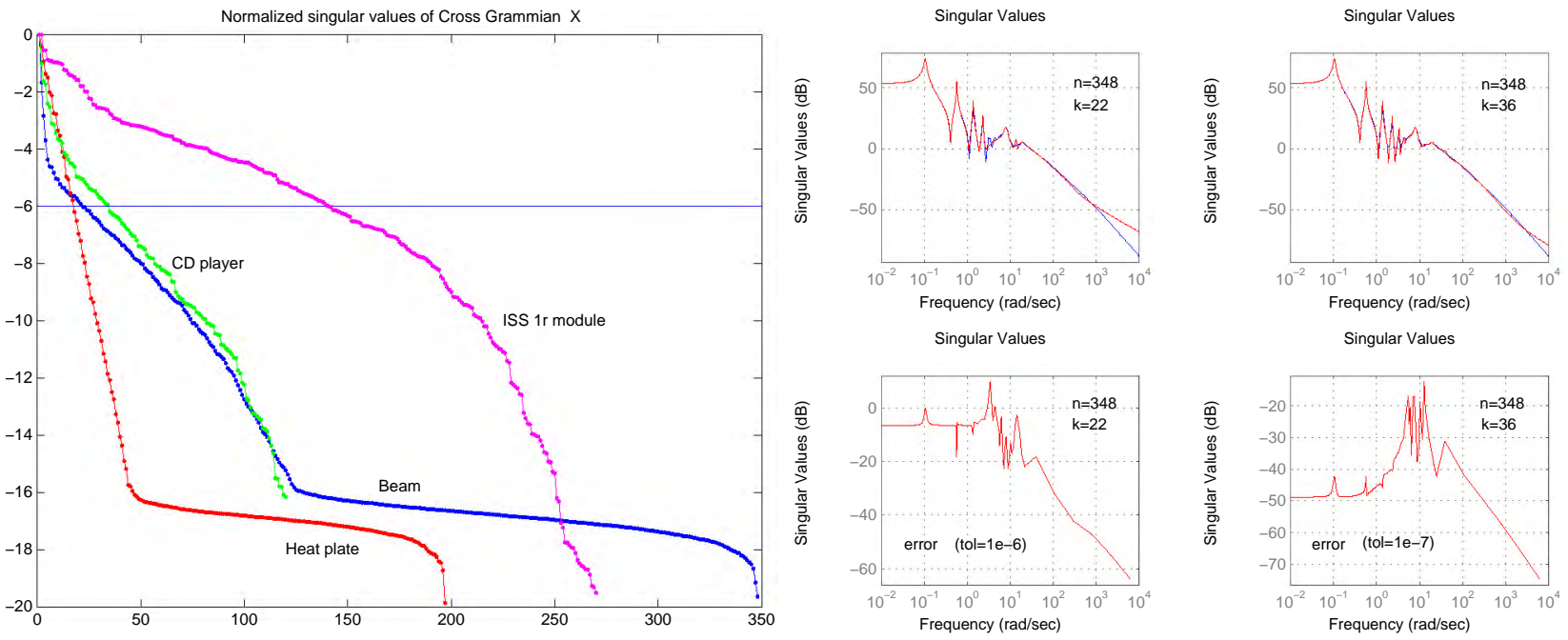

Figure 3: Hankel singular values of 4 examples; Clamped Beam Displacement

In figure 3, the plot on the left-hand side compares the Hankel singular values of four systems: a finite-element discretization of a heat plate, a finite-element discretization of a clamped beam, the model of a component of a CD player, and a model of the flex modes of the 1r module of the ISS (International Space Station). It follows from this plot that for a give tolerance, the ISS model is the hardest to approximate, as its Hankel singular values decay the least rapidly.

The right-hand side subplot shows results for a finite element calculation of displacement and stress in the clamped beam with Rayleigh (or proportional) damping. The input is a force at the unclamped end, while the output is displacement. The figure shows a Bode plot of the response of the original system which is of order 348 (solid line) and response of the reduced order model of order 48 (dashed line). The order of the reduced model is automatically determined once the error requirement is specified.

\section{$5 \quad$ Unifying features and conclusions}

The unifying feature of all model reduction methods is that the main operation is a projection. Given $\boldsymbol{\Sigma}$ (3), and a projection $\pi=\pi_{L} \pi_{R}$ (i.e. $\pi_{R} \pi_{L}=I_{k}$ ) a reduced order model $\hat{\boldsymbol{\Sigma}}$ is obtained by projecting $\boldsymbol{\Sigma}$ as follows:

$$
\hat{\mathbf{\Sigma}}=\left(\begin{array}{c|c}
\pi_{L} A \pi_{R} & \pi_{L} B \\
\hline C \pi_{R} &
\end{array}\right): \frac{d \hat{x}}{d t}=\left[\pi_{L} A \pi_{R}\right] \hat{x}+\left[\pi_{L} B\right] u, y=\left[C \pi_{R}\right] \hat{x}
$$

The quality of the reduced model is measured in terms of the frequency response

$$
H(j \omega)=D+C(j \omega I-A)^{-1} B
$$

Choices of projectors for the reduction methods discussed above are:

1. Balanced truncation: Project onto the dominant eigenspace of $\mathcal{P} \mathcal{Q}$, where $\mathcal{P}, \mathcal{Q}$ are the grammians.

2. Optimal Hankel norm approximation: Solve for grammians. Embed in a lossless transfer function and project onto its stable eigenspace.

3. Krylov-based approximation: Project onto controllability and/or observability spaces, i.e. $\mathcal{R}$ and $\mathcal{O}$. 
4. New method [4]: Project onto the dominant eigenspace of the best rank-k approximant $X_{k}$ of the cross grammian $X$.

Complexity issues [12]. The major costs associated with computing balanced and Hankel norm approximants are: approximately $30 n^{3}$ for computing the grammians and approximately $25 n^{3}$ for performing the balancing. For rational Krylov at $k$ points, $\frac{2}{3} k n^{3}$ operations are needed. Of course various iterative methods (sign, disc, Smith) can accelerate the computation of grammians in particular on parallel machines. For approximate or sparse decompositions we need $c_{1} \alpha k n$ operations to compute the grammians and $\mathcal{O}\left(n^{3}\right)$ operations for embedding or balancing. The corresponding number for rational Krylov is $c_{2} k \alpha n$, where $k$ is the number od expansion points and $\alpha$ is the average number of non-zero elements per row in $A$.

Conclusions. We have outlined the current trends in the computation of reduced-order models for large-scale systems. The SVD-based methods and the Krylov-based methods each enjoy disjoint sets of advantages. Therefore a third approach referred to as SVD-Krylov, has emerged which aims at combining the best attributes of these two methods.

\section{References}

[1] V.M. Adamjan, D.Z. Arov, and M.G. Krein, Analytic properties of Schmidt pairs for a Hankel operator and the generalized Schur-Takagi problem, Math. USSR Sbornik, 15: 31-73 (1971).

[2] V.M. Adamjan, D.Z. Arov, and M.G. Krein, Infinite block Hankel matrices and related extension problems, American Math. Society Transactions, 111: 133-156 (1978).

[3] A.C. Antoulas, Recursive modeling of discrete-time series, in P. Van Dooren and B.F. Wyman editors, IMA volume on Linear Algebra for Control, pp. 1-22, (1993).

[4] A.C. Antoulas and D.C. Sorensen, Projection methods for balanced model reduction, Technical Report ECE-CAAM Depts, Rice University, March 2001.

[5] A.C. Antoulas and R.H. Bishop, Continued-fraction decomposition of linear systems in the state space, Systems and Control Letters, 9: 43-53 (1987).

[6] A.C. Antoulas and J.C. Willems, A behavioral approach to linear exact modeling, IEEE Trans. Automatic Control, AC-38: 1776-1802, (1993).

[7] A.C. Antoulas, E.J. Grimme, and D. Sorensen, On behaviors, rational interpolation and the Lanczos algorithm, Proc. IFAC World Congress, San Francisco (1996).

[8] A.C. Antoulas, Approximation of linear operators in the 2-norm, Linear Algebra and its Applications, Special Issue on Challenges in Matrix Theory, (1998).

[9] A.C. Antoulas, Approximation of linear dynamical systems, in the Wiley Encyclopedia of Electrical and Electronics Engineering, edited by J.G. Webster, volume 11: 403-422 (1999).

[10] A.C. Antoulas, On eigenvalues and singular values of linear dynamical systems, Proc. XVI Housholder Symposium, Chateau Whistler (1999).

[11] A.C. Antoulas and D.C. Sorensen, Lyapunov, Lanczos and Inertia, Linear Algebra and Its Applications, LAA (2001).

[12] A.C. Antoulas and P.M. van Dooren, Approximation of large-scale dynamical systems, Notes of a Short Course given at the SIAM Annual Meeting, Puerto Rico (2000). (Notes available from the authors' websites.)

[13] A.C. Antoulas, Approximation of large-scale dynamical systems, SIAM Press (to appear). 
[14] A.C. Antoulas, J.A. Ball, J. Kang, and J.C. Willems, On the solution of the minimal rational interpolation problem, Linear Algebra and its Applications, 137/138: 511-573 (1990).

[15] A.C. Antoulas and B.D.O. Anderson, On the problem of stable rational interpolation, Linear Algebra and its Applications, 122-124: 301-329 (1989).

[16] W.E. Arnoldi, The principle of minimized iterations in the solution of the matrix eigenvalue problem, Quart. Applied Math., 9: 17-29 (1951).

[17] K.E. Avrachenkov and J.B. Lasserre, Analytic perturbation of Sylvester matrix equations, Proc. IEEE Conf. Decision and Control, Sydney, December 2000.

[18] Z. Bai, Q. Ye, Error estimation of the Padé approximation of transfer functions via the Lanczos process, Electron. Transactions Numer. Analysis, 7: 1-17 (1998).

[19] Z. Bai, P. Feldmann, and R.W. Freund, Stable and passive reduced-order models based on partial Padé approximation via the Lanczos process, Numerical Analysis Manuscript 97-3-10, Bell Laboratories, Murray Hill (1997).

[20] R.H. Bartels and G.W. Stewart, Solution of the matrix equation $A X+X B=C$, Communications of the ACM, 15: 820-826 (1972).

[21] G. Berkooz, P. Holmes, and J.L. Lumley, Coherent structures, dynamical systems and symmetry, Cambridge Monographs on Mechanics, Cambridge University Press (1996).

[22] D.L. Boley and G.H Golub, The Lanczos algorithm and controllability, Systems and Control Letters, 4: $317-324$ (1984).

[23] D.L. Boley, Krylov space methods on state-space control models, Circuits, Systems, and Signal Processing, 13: 733758 (1994).

[24] M.T. Chu, R.E. Funderlic, and G.H. Golub, A rank-one reduction formula and its applications to matrix factorizations, SIAM Review, 37: 512-530 (1995).

[25] B.N. Datta and Y. Saad, Arnoldi methods for large Sylvester-like observer matrix equations and an associated algorithm for partial spectrum assignment, Lin. Alg. Appl., vol. 154-156, pp. 225-244, (1991).

[26] E. de Souza and S.P. Bhattacharyya, Controllability, observability and the solution of $A X-X B=C$, Linear Algebra and its Applications, 39: 167-188 (1981).

[27] D.F. Enns, Model reduction with balanced realizations: An error bound and frequency weighted generalization, Proc. of the IEEE Conference on Decision and Control, 127-132 (1984).

[28] K.V. Fernando and H. Nicholson, On the structure of balanced and other principal representations of SISO systems, IEEE Trans. Automatic Control, AC-28: 228-231 (1983).

[29] P. Feldmann and R.W. Freund, Efficient linear circuit analysis by Padé approximation via the Lanczos process, IEEE Trans. Computer-Aided Design, 14: 639-649 (1995).

[30] R.W. Freund, Reduced-order modeling techniques based on Krylov subspaces and their use in circuit simulation, in Applied and Computational Control, Signals, and Circuits, Birkhäuser, vol. 1, pp. 435-498 (1999).

[31] P.A. Fuhrmann, A polynomial approach to Hankel norm and balanced approximations, Linear Algebra and its Applications, 146: 133-220 (1991).

[32] K. Gallivan, E.J. Grimme, and P. Van Dooren, Asymptotic waveform evaluation via a restarted Lanczos method, Applied Math. Letters, 7: 75-80 (1994).

[33] K. Gallivan, E. Grimme and P. Van Dooren, Padé Approximation of large-scale dynamic systems with Lanczos methods, Proc. 33rd IEEE Conf. on Decision and Control, (Lake Buena Vista, FL), (1994). 
[34] K. Gallivan, E. Grimme and P. Van Dooren, A rational Lanczos algorithm for model reduction, CSRD Report No. 1411, University of Illinois at Urbana-Champaign, IL, (1995).

[35] A.R. Ghavimi and A.J. Laub, Numerical methods for nearly singular constrained matrix Sylvester equations, SIAM J. Matrix Anal. Appl., 17: 212-221 (1996).

[36] K. Glover, All optimal Hankel-norm approximations of linear multivariable systems and their $L^{\infty}$-error bounds, International Journal of Control, 39: 1115-1193 (1984).

[37] W.B. Gragg, The Padé table and its relation to certain algorithms of numerical analysis, SIAM Review, 14: 1-62 (1972).

[38] W.B. Gragg and A. Lindquist, On the partial realization problem, Linear Algebra and Its Applications, Special Issue on Linear Systems and Control, 50: 277-319 (1983).

[39] E.J. Grimme, Krylov Projection Methods for Model Reduction, Ph.D. Thesis, ECE Dept., U. of Illinois, UrbanaChampaign,(1997).

[40] E.J. Grimme, D.C. Sorensen, and P. Van Dooren, Model reduction of state space systems via an implicitly restarted Lanczos method, Numerical Algorithms, 12: 1-31 (1995).

[41] G.H. Golub and C.F. Van Loan, Matrix computations, The Johns Hopkins University Press (1989).

[42] M.H. Gutknecht, The Lanczos process and Padé approximation, Proc. Cornelius Lanczos Intl. Centenary Conference, edited by J.D. Brown et al., pages 61-75, SIAM, Philadelphia (1994).

[43] M.T. Heath, A.J. Laub, C.H. Paige, and R.C. Ward, Computing the singular value decomposition of a product of two matrices, SIAM J. Sci. Stat. Computing, 7: 1147-1159 (1986).

[44] A.S. Hodel, R.B. Tenison, and K. Poola, Numerical solution of large Lyapunov equations by approximate power iteration, Linear Algebra Appl., 236: 205-230 (1996).

[45] A.S. Hodel and P. Misra, Least-squares approximate solution of overdetermined Sylvester equations, SIAM J. Matrix Anal. Appl., 18: 279-290 (1997).

[46] D. Hu and L. Reichel, Krylov-subspace methods for the Sylvester equation, Linear Alg. and Appl., 172, 283-313, (1992).

[47] L. Hubert, J. Meuleman, and W. Heiser, Two purposes for matrix factorization: A historical appraisal, SIAM Review, 42: 68-82 (2000).

[48] I.M. Jaimoukha, E.M. Kasenally, Implicitly restarted Krylov subspace methods for stable partial realizations, SIAM J. Matrix Anal. Appl., 18: 633-652 (1997).

[49] R.E. Kalman, On partial realizations, transfer functions, and canonical forms, Acta Polyt. Scand. Ma., 31: 9-32 (1979).

[50] M. Kamon, F. Wang, and J. White, Generating nearly optimal compact models from Krylov-subspace based reducedorder models, IEEE Trans. Circuits and Systems-II: Analog and digital signal processing, 47: 239-248 (2000).

[51] S.W. Kim, B.D.O. Anderson and A.G. Madievski, Error bound for transfer function order reduction using frequency weighted balanced truncation, Systems and Control Letters, 24: 183-192 (1995).

[52] S. Lall, J.E. Marsden, and S. Glavaski, Empirical model reduction of controlled nonlinear systems, Technical Report CIT-CDS-98-008, California Institute of Technology, (1998).

[53] P. Lancaster, Explicit solutions of matrix equations, SIAM Review, 12: 544-566 (1970). 
[54] C. Lanczos, An iteration method for the solution of the eigenvalue problem of linear differential and integral operators, J. Res. Nat. Bureau Stan., 45 255-282, (1950).

[55] A.J. Laub, Computation of balancing transformations, Proc. Joint Autom. Control Conf., San Francisco (1980).

[56] A.J. Laub, M.T. Heath, C.C. Paige, and R.C. Ward, Computation of system balancing transformations and other applications of simultaneous diagonalization algorithms, IEEE Trans. Automatic Control, AC-32: 115-121 (1987).

[57] R.B. Lehoucq, D.C. Sorensen, and C. Yang, ARPACK: User's Guide, SIAM (1998).

[58] R.B. Lehoucq and A.G. Salinger, Large-scale eigenvalue calculations for stability analysis of steady flows on massively parallel computers, Technical Report, Sandia (2000).

[59] J. Li, F. Wang, J. White, An efficient Lyapunov equation-based approach for generating reduced-order models of interconnect, Proc. 36th IEEE/ACM Design Automation Conference, New Orleans, LA, (1999).

[60] B.C. Moore, Principal component analysis in linear systems: Controllability, observability and model reduction, IEEE Trans. Automatic Control, AC-26: 17-32 (1981).

[61] R. Ober, Balanced parametrization of classes of linear systems, SIAM J. Control and Optimization, 29: 1251-1287 (1991).

[62] G. Obinata and B.D.O. Anderson, Model reduction for control system design, Springer Verlag (2000).

[63] T. Penzl, A cyclic low rank Smith method for large sparse Lyapunov equations, SIAM J. Sci. Comp. (to appear).

[64] L.T. Pillage and R.A. Rohrer, Asymptotic waveform evaluation for timing analysis, IEEE Trans. Comp. Aided Design, 9: 352-366 (1990).

[65] T.D. Romo, J.B. Clarage, D.C Sorensen, and G.N. Phillips Jr., Automatic identification of discrete substates in proteins: Singular value decomposition analysis of time-averaged crystallographic refinements, Proteins: Structure, Function, and Genetics, 22: 311-321 (1995).

[66] W.E. Roth, The equation $A X-Y B=C$ and $A X-X B=C$ in matrices, Proc. Amer. Math. Soc., 3: 392-396 (1952).

[67] A. Ruhe, Rational Krylov sequence methods for eigenvalue computation, Linear Algebra and Appl., vol. 58, pp. 391405, (1984).

[68] Y. Saad, Numerical solution of large Lyapunov equations, in Signal Processing, Scattering and Operator Theory, and Numerical Methods, Proc. MTNS-89, 3: 503-511, Birkhäuser (1990).

[69] V. Simoncini, On the numerical solution of $A X-X B=C$, BIT, 36: 814-830 (1996).

[70] D.C. Sorensen, Implicit application of polynomial filters in a k-step Arnoldi method, SIAM J. Matrix Anal. Applic., 13: $357-385$ (1992).

[71] D.C. Sorensen, Implicitly restarted Arnoldi/Lanczos methods and large-scale SVD applications, in SVD and Signal Processing III, edited by M. Moonen and B. de Moor, Elsevier (1995).

[72] D.C. Sorensen, Lecture notes on numerical methods for large scale eigenvalue problems, Draft of Lectures delivered at the Royal Institute of Technology KTH, Stockholm, Spring 1999.

[73] D. Stroobandt, Recent advances in system-level interconnect prediction, IEEE Circuits and Systems Newsletter, 11: 4-20 (2000).

[74] P. Van Dooren, The Lanczos algorithm and Padé approximations, Short Course, Benelux Meeting on Systems and Control, (1995). 
[75] P.M. Van Dooren, Gramian based model reduction of large-scale dynamical systems, in Numerical Analysis 1999, Chapman and Hall, pp. 231-247, CRC Press, London, 2000.

[76] A. Varga, Balancing-free square-root algorithms for computing singular perturbation approximations, Proc. 30th IEEE CDC, Brighton, UK, pages 1062-1065 (1991).

[77] A. Varga, Enhanced modal approach for model reduction, Mathematical Modelling of Systems, 1: 91-105 (1995).

[78] A. Varga, Computation of normalized coprime factorizations of rational matrices, Systems and Control Letters, 33 : 37-45 (1998).

[79] A. Varga, Model reduction routines for SLICOT, Niconet Report 1999-8, (1999). Report available from: ftp://wgs . esat.kuleuven.ac.be/pub/WGS/REPORTS/NIC1999-8.ps.Z

[80] A. Varga, Balanced truncation model reduction of periodic systems, Proc. IEEE CDC, Session WeP05, Sydney, December 2000.

[81] K. Zhou, Frequency-weighted $\mathcal{L}_{\infty}$ norm and optimal Hankel norm model reduction, IEEE Trans. Aut. Control, AC-40: 1687-1699 (1995).

[82] S. Volkwein, Proper orthogonal decomposition and singular value decomposition, Technical Report SFB-153, Institut für Mathematik, Universität Graz (1999).

[83] K. Zhou, A new balanced realization and model reduction method for unstable systems, Proc. 14th IFAC World Congress, Beijing, Volume D-2b-04-4: 123-128 (1999). 